\title{
BIFURCATION OF LOCALIZED DISTURBANCES IN A MODEL BIOCHEMICAL REACTION*
}

\author{
JAMES A. BOA AND DONALD S. COHEN†
}

\begin{abstract}
Asymptotic solutions are presented to the nonlinear parabolic reaction-diffusion equations describing a model biochemical reaction proposed by I. Prigogine. There is a uniform steady state which, for certain values of the adjustable parameters, may be unstable. When the uniform solution is slightly unstable, the two-timing method is used to find the bifurcation of new solutions of small amplitude. These may be either nonuniform steady states or time-periodic solutions, depending on the ratio of the diffusion coefficients. When one of the parameters is allowed to depend on space and the basic state is unstable, it is found that the nonuniform steady state which is approached may show localized spatial oscillations. The localization arises out of the presence of turning points in the linearized stability equations.
\end{abstract}

1. Introduction. Under certain circumstances the usual equilibrium state of a chemical reaction may be unstable with respect to small changes in the concentrations, or to other perturbations. Experimental work ([2], [7], [8]) has shown that in the subsequent development of the reaction, there may be established selfsustaining oscillations in concentration, concentration waves, or new steady states that are not uniform. These phenomena have been thought of interest because of their possible implications in the study of circadian rhythms, short-term memory, cardiac fibrillation, and morphogenesis. (The list is not exhaustive.) However, the prediction and quantitative description of any such phenomena clearly require a detailed knowledge of the reaction mechanism; this is in most cases lacking. Accordingly, it is necessary to study model reactions with known kinetics, to see whether the solutions of the rate equations exhibit any of the phenomena that are found in the laboratory. One model reaction that has received considerable attention is the reaction $A+B \rightarrow D+E$ according to the sequence

$$
\begin{aligned}
A & \rightarrow X \\
2 X+Y & \rightarrow 3 X \\
B+X & \rightarrow Y+D \\
X & \rightarrow E .
\end{aligned}
$$

This model is due to I. Prigogine.

In $\S 2$ of this paper we derive the rate equations for this reaction and report the numerical results of Herschkowitz-Kaufman and Nicolis [3], who find that when the basic steady state of the reaction loses its stability, the resulting disturbance can show an interesting localization effect. In $\S 3$ we consider the

* Received by the editors August 5, 1974.

† Department of Applied Mathematics, California Institute of Technology, Pasadena, California 91109. The first author is now at Department of Mathematics and Center for Mathematical Methods in the Social, Behavioral, Biological, and Health Sciences, State University of New York at Buffalo, Amherst, New York 14226. This work was supported in part by the U.S. Army Research Office (Durham) under Contract DAHC-04-68-C-0006 and by the National Science Foundation under Grant GP 18471. 
linearized stability analysis of the basic steady state in the case that it is uniform, and in $\S 4$ we consider the bifurcation of nonuniform steady states from the uniform state. In $\S 5$ we show that the localization of the disturbance described in $\S 2$ is a result of the presence of turning points in the linearized stability equations.

2. Governing equations. In deriving the rate equations describing Prigogine's model reaction, the simplest possible assumption is made about the rate of reaction: namely, that it is proportional to the correct product of the concentrations. Thus the presence of the autocatalytic step $2 X+Y \rightarrow 3 X$ makes the rate equations nonlinear, since the rate of production of reactant $X$ from this step is proportional to $[X]^{2}[Y]$. A further simplifying assumption is made by setting all the rate constants equal to one. This means in particular that temperature variations have been completely neglected; such an assumption seems not unreasonable, since chemical reactions going on in living tissue cannot be very exothermic. Also, it is supposed that there is no convective motion of the reactants, and back reactions are neglected. Then the conservation equations for the concentrations $A, B, X, Y, D, E$ are

$$
\begin{aligned}
& \frac{\partial A}{\partial t}=-A+D_{A} \nabla^{2} A \\
& \frac{\partial X}{\partial t}=A+X^{2} Y-B X-X+D_{X} \nabla^{2} X \\
& \frac{\partial Y}{\partial t}=-X^{2} Y+B X+D_{Y} \nabla^{2} Y, \\
& \frac{\partial D}{\partial t}=B X+D_{D} \nabla^{2} D \\
& \frac{\partial E}{\partial t}=X+D_{E} \nabla^{2} E .
\end{aligned}
$$

We have written $X$ instead of $[X]$ for the concentration of reactant $X$, and so forth, since there can be no risk of ambiguity. The $D$ 's are diffusion coefficients. It will be noticed that the equation for $A$ is not coupled to the rest so that it may be solved first. Further, the equations for $D$ and $E$ become merely inhomogeneous linear equations once those for $B, X$, and $Y$ have been solved. Actually HerschkowitzKaufman and Nicolis [3] make the further assumption that the concentration of $B$ is somehow maintained uniform (alternatively, $D_{B}=\infty$ ) so that $B$ appears only parametrically in the equations for $X$ and $Y$. Also, they take $D_{A}$ to be fairly large so that $A$ relaxes fairly quickly to its steady state. They then substitute this steady state value of $A$ into the equation for $X$, and consider the reaction to be taking place in an infinite slab of width 1 . The differential equations which they consider are thus

$$
\begin{aligned}
& \frac{\partial X}{\partial t}=A(r)-(B+1) X+X^{2} Y+D_{X} \frac{\partial^{2} X}{\partial r^{2}} \\
& \frac{\partial Y}{\partial t}=B X-X^{2} Y+D_{Y} \frac{\partial^{2} Y}{\partial r^{2}}
\end{aligned}
$$


where $r$ is the spatial coordinate and

$$
A(r)=\bar{A} \operatorname{sech} \frac{1}{\sqrt{D_{A}}} \cosh \frac{r-\frac{1}{2}}{\sqrt{D_{A}}} .
$$

They pick $D_{A}=197 \times 10^{-3}, D_{X}=1.05 \times 10^{-3}, \bar{A}=14$ and allow $B$ and $D_{Y}$ to be free parameters. The boundary conditions are that $X(0, t)=X(1, t)=\bar{A}$ and $Y(0, t)=Y(1, t)=B / \bar{A}$.

In the limit $D_{A}=\infty$, so that $A(r) \equiv$ const. $=\bar{A}$, there is a uniform steady state $X(r, t) \equiv \bar{A}, Y(r, t) \equiv B / \bar{A}$. When $A(r)$ is not constant, and $D_{X}$ and $D_{Y}$ are small, there is a steady state $X \sim A(r)+O\left(D_{X}, D_{Y}\right), Y \sim B / A(r)+O\left(D_{X}, D_{Y}\right)$. This might be called the basic steady state. (See Fig. 2.1.) It should be noticed that the

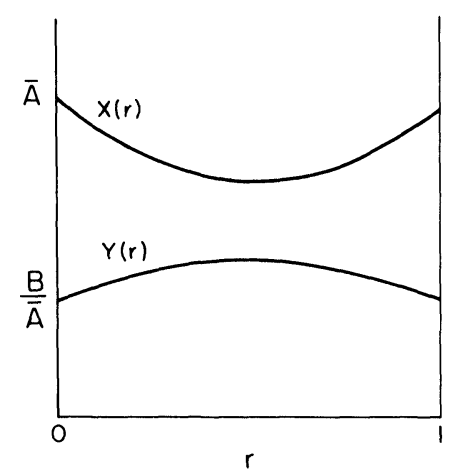

FIG. 2.1. The basic steady state

boundary conditions have been chosen in such a manner that the basic state satisfies them. Thus, no boundary layers occur at the edges of the slab, even though $D_{X}$ and $D_{Y}$ are small and multiply the highest order derivatives. Herschkowitz-Kaufman and Nicolis find that for certain values of the parameters $B$ and $D_{Y}$ the basic state is unstable against small perturbations in the initial conditions. The subsequent development of the system can lead either to a new steady state which shows spatial oscillations ("dissipative structure") or to propagating waves of concentration. Figure 2.2 shows the dissipative structure

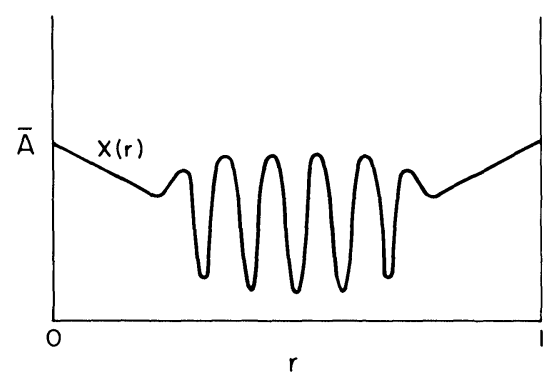

FIG. 2.2. A localized dissipative structure 
that is found for the choices $D_{Y}=5.25 \times 10^{-3}, B=26$. This picture has been copied from the paper of Herschkowitz-Kaufman and Nicolis. It is seen that the disturbance from the basic state is confined to the center of the slab so that the instability of the basic state can be considered local. The localization is clearly due to the nonuniform distribution of reactant $A$.

In attempting in $\S 5$ to explain the localization we shall take advantage of the fact that $D_{X} \ll 1$ and $D_{Y} \ll 1$. The natural length scale on which $X$ may be expected to vary appreciably is $D_{X}^{1 / 2}$, whereas $A$ varies on a length scale of $D_{A}^{1 / 2}$. The ratio of these quantities is

$$
\left(D_{X} / D_{A}\right)^{1 / 2}=\left(1.05 \times 10^{-3} / 197 \times 10^{-3}\right)^{1 / 2} \approx 1 / 14,
$$

so that variations in $A$ can be considered slow compared to those in $x$. The case in which $A$ is uniformly distributed is also of interest, since solutions can exhibit both nonuniform steady states and waves, although not localization.

3. Stability of the basic state. The linearized stability analysis of the basic steady state (or uniform steady state in the case that $A(r)$ is uniformly distributed) may be accomplished by standard methods. One writes $X=X_{0}(r)+u(r, t)$, $Y=Y_{0}(r)+v(r, t)$, substitutes into (2.1) and (2.2), and omits terms quadratic or higher in $u$ and $v$ in the resulting differential equations. The linearized stability equations are therefore

$$
\begin{aligned}
& \frac{\partial u}{\partial t}=\left[-(B+1)+2 X_{0} Y_{0}\right] u+X_{0}^{2} v+D \frac{\partial^{2} u}{\partial r^{2}} \\
& \frac{\partial v}{\partial t}=\left[B-2 X_{0} Y_{0}\right] u-X_{0}^{2} v+\vartheta D \frac{\partial^{2} v}{\partial r^{2}}
\end{aligned}
$$

together with the boundary conditions $u(0, t)=u(1, t)=v(0, t)=v(1, t)=0$.

Solutions to this boundary value problem are found by separation of variables. One writes

$$
\mathbf{u}=\left[\begin{array}{l}
u(r, t) \\
v(r, t)
\end{array}\right]=e^{\sigma t} \boldsymbol{\phi}=e^{\sigma t}\left[\begin{array}{l}
\phi(r) \\
\psi(r)
\end{array}\right]
$$

then $\sigma$ must be an eigenvalue and $\phi$ an eigenfunction of the nonself-adjoint problem

$$
\begin{aligned}
& \sigma \phi=\left[-(B+1)+2 X_{0} Y_{0}\right] \phi+X_{0}^{2} \psi+D \phi^{\prime \prime}, \\
& \sigma \psi=\left[B-2 X_{0} Y_{0}\right] \phi-X_{0}^{2} \psi+\vartheta D \psi^{\prime \prime}, \\
& \phi(0)=\phi(1)=\psi(0)=\psi(1)=0 .
\end{aligned}
$$

The solution to (3.1) and (3.2) is then given by the expansion

$$
\mathbf{u}=\operatorname{Re} \sum c_{n} e^{\sigma_{n} t} \boldsymbol{\phi}_{n},
$$

where the $\sigma_{n}$ are the eigenvalues of the boundary value problem (3.4)-(3.6), the $\boldsymbol{\phi}_{n}$ are the corresponding eigenfunctions, and the coefficients $c_{n}$ are found from the initial conditions. The basic state is called stable if the real parts of all the 
eigenvalues are negative, unstable if some eigenvalues have positive real parts, and "neutrally stable" if the greatest real part for any eigenvalue is zero.

If $A(r)$ is uniformly distributed, then $X_{0}(r)=A$ and $Y_{0}(r)=B / A$, and (3.4) and (3.5) become

$$
\begin{aligned}
& \sigma \phi=(B-1) \phi+A^{2} \psi+D \phi^{\prime \prime}, \\
& \sigma \psi=-B \phi-A^{2} \psi+\vartheta D \psi^{\prime \prime} .
\end{aligned}
$$

Since this system has constant coefficients, the eigenvalues and eigenfunctions (which are trigonometric sines) can be found exactly.

Substitution of $\phi_{n}(r)=\sin n \pi r, \psi_{n}(r)=M_{n} \sin n \pi r$ (with $M_{n}$ to be determined) into (3.7) and (3.8) yields the homogeneous system

$$
\left[\begin{array}{cc}
\sigma-(B-1)+n^{2} \pi^{2} D & -A^{2} \\
B & \sigma+A^{2}+n^{2} \pi^{2} \vartheta D
\end{array}\right]\left[\begin{array}{l}
\phi \\
\psi
\end{array}\right]=\left[\begin{array}{l}
0 \\
0
\end{array}\right] .
$$

There will be nontrivial solutions to (3.9) only if the determinant of the coefficients is zero; that is, if

$$
\begin{aligned}
\sigma^{2}+\sigma[-B+1 & \left.+A^{2}+n^{2} \pi^{2} D(1+\vartheta)\right]+A^{2} B \\
& +\left[A^{2}+n^{2} \pi^{2} \vartheta D\right]\left[-(B-1)+n^{2} \pi^{2} D\right]=0 .
\end{aligned}
$$

The eigenvalue $\sigma$ with the greatest real part does not necessarily correspond to $n=1$ but depends on the parameters $\vartheta$ and $B$. Thus, when the uniform solution loses its stability, the first eigenfunction of (3.4)-(3.6) that is excited is not necessarily a multiple of $\sin \pi r$.

It will be convenient to change the notation slightly. Associated with each value of $n$ there will be two eigenvalues, the roots of (3.10). We call them $\sigma_{n}^{+}$and $\sigma_{n}^{-}$, according to the definition

$$
\begin{aligned}
\sigma_{n}^{ \pm}=\frac{1}{2}\left\{B-1-A^{2}-\right. & n^{2} \pi^{2} D(1+\vartheta) \\
\pm & {\left.\left[\left[B-1+A^{2}+n^{2} \pi^{2} D(\vartheta-1)\right]^{2}-4 A^{2} B\right]^{1 / 2}\right\} }
\end{aligned}
$$

The eigenfunction corresponding to $\sigma_{n}^{ \pm}$will be called

$$
\boldsymbol{\phi}_{n}^{ \pm}=\left[\begin{array}{c}
\sin n \pi r \\
M_{n}^{ \pm} \sin n \pi r
\end{array}\right] \text {. }
$$

The coefficient $M_{n}^{ \pm}$is given from (3.9) by

$$
\sigma_{n}^{ \pm}-(B-1)+n^{2} \pi^{2} D-A^{2} M_{n}^{ \pm}=0 .
$$

The curve of neutral stability (the stability boundary) of the uniform solution in the $(\vartheta, B)$-plane is shown in Fig. 3.2. It is found by considering the curves $\operatorname{Re} \sigma_{n}^{+}=0$; we now do this. (It is seen from (3.11) that $\operatorname{Re} \sigma_{n}^{+} \geqq \operatorname{Re} \sigma_{n}^{-}$, with equality if $\sigma_{n}^{+}$and $\sigma_{n}^{-}$are complex.) If $\sigma_{n}^{+}$is complex, then the curve $\operatorname{Re} \sigma_{n}^{+}=0$ is the straight line

$$
B=1+A^{2}+n^{2} \pi^{2} D(1+\vartheta),
$$

while if $\sigma_{n}^{+}$is real, the curve $\operatorname{Re} \sigma_{n}^{+}=0$ is the rectangular hyperbola $H_{n}$, given by

$$
B=1+n^{2} \pi^{2} D+\frac{A^{2}}{\vartheta}\left\{1+\frac{1}{n^{2} \pi^{2} D}\right\}
$$




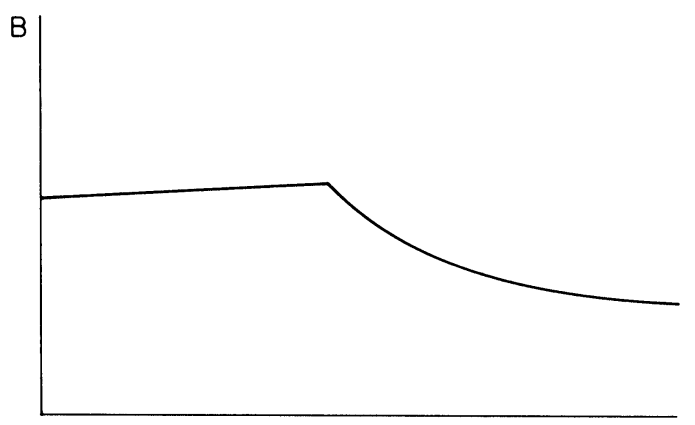

FIG. 3.1. The curve $\operatorname{Re}\left(\sigma_{n}^{+}\right)=0$ for a typical value of $n$

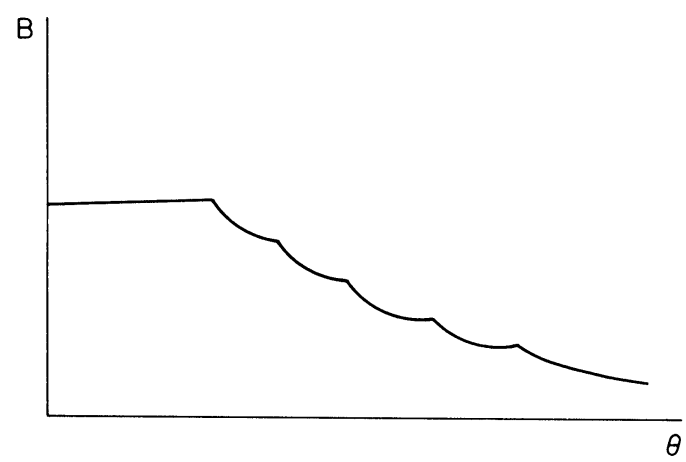

FIG. 3.2. Neutral stability curve of the uniform solution

If $\vartheta=0, \sigma_{n}^{+}$is complex as its real part changes sign, so that the curve $\operatorname{Re} \sigma_{n}^{+}=0$ is given by (3.13) for small $\vartheta$. The straight line (3.13) ceases to represent $\operatorname{Re} \sigma_{n}^{+}=0$ after it intersects the hyperbola (3.14), as $\sigma_{n}^{+}$is real as its real part changes sign for values of $\vartheta$ larger than the one at the point of intersection. Thus the curve $\operatorname{Re} \sigma_{n}^{+}=0$ in the $(\vartheta, B)$-plane is as sketched in Fig. 3.1.

The curve of neutral stability (the stability boundary) in the $(\vartheta, B)$-plane for the uniform solution is found by superposing all the curves $\operatorname{Re} \sigma_{n}^{+}=0$ and determining which of them lie the lowest. It is clear from (3.13) that the straight line portion of the curve $\operatorname{Re} \sigma_{1}^{+}=0$ lies the lowest for sufficiently small $\vartheta$. It will continue to represent the curve of neutral stability until it intersects one of the hyperbolas given by (3.14). Which one it is depends on the parameters $A$ and $D$. It is seen from (3.14) that each hyperbola intersects all the rest; $H_{1}$ lies the lowest for sufficiently large $\vartheta$, and as $\vartheta$ decreases, one finds that portions of $\mathrm{H}_{2}$, then $\mathrm{H}_{3}$ and so forth, lie the lowest in the $(\vartheta, B)$-plane. The point $K_{n}$ where $H_{n}$ and $H_{n+1}$ intersect is at

$$
\vartheta=\frac{A^{2}}{n^{2}(n+1)^{2}\left(\pi^{2} D\right)^{2}}
$$


The curve of neutral stability for the uniform solution is therefore scallopshaped. A typical case is sketched in Fig. 3.2. Below the curve the uniform solution is stable, and above it unstable. The curve of neutral stability for the basic steady state in the case that $A(r)$ is not constant is presumably similar.

4. Bifurcation of nonuniform steady states. When the uniform state is unstable, solutions starting with initial conditions near the uniform state tend away from it. The time development of solutions can be calculated asymptotically when the point $\vartheta, B$ is only slightly above the neutral stability curve in the $(\vartheta, B)$-plane. The technique we shall use is the two-timing method of Matkowsky [5], which is worked out in detail by Kogelman and Keller [4]. It is found that if the point $\vartheta, B$ is above the straight line portion of the neutral stability curve, so that the eigenfunctions $\phi_{1}^{+}$and $\phi_{1}^{-}$are excited first, the solution tends to a timeperiodic disturbance of small amplitude. If the point $\vartheta, B$ is above the curved portions of the neutral stability curve, the situation that is considered in this section, the solution for certain initial conditions tends to a nonuniform steady state of small amplitude. This steady state is (to first order in the perturbation parameter) a multiple of the eigenfunction $\phi_{N}^{+}$that is first excited. The value of $N$ that applies on which of the hyperbolas $H_{n}$ the point $\vartheta, B$ lies above, and therefore depends on $\vartheta$. There is also an exceptional case in which $\vartheta, B$ lies above one of the intersection points $K_{N}$. In that case, both $\boldsymbol{\phi}_{N}^{+}$and $\boldsymbol{\phi}_{N+1}^{+}$are excited.

Nicolis and Auchmuty [6] have also considered the bifurcation of nonuniform steady states. However, their technique does not reveal how these states depend on the initial conditions, and they omit the exceptional cases. While we include the results here for the sake of completeness, we omit most of our calculations since the technique follows that of Kogelman and Keller [4] exactly and they are given in detail by Boa [9].

When $A$ is uniform, equations (2.1) and (2.2) become

$$
\begin{aligned}
& \frac{\partial X}{\partial t}=A-(B+1) X+X^{2} Y+D \frac{\partial^{2} X}{\partial r^{2}} \\
& \frac{\partial Y}{\partial t}=B X-X^{2} Y+\vartheta D \frac{\partial^{2} Y}{\partial r^{2}}
\end{aligned}
$$

the boundary conditions are $X(0, t)=X(1, t)=A$ and $Y(0, t)=Y(1, t)=B / A$. We suppose that initial conditions are given of the form

$$
\begin{aligned}
& X(r, 0)=A+h(r, \epsilon), \\
& Y(r, 0)=B / A+k(r, \epsilon),
\end{aligned}
$$

where $h(r, \epsilon)$ and $k(r, \epsilon)$ are functions which satisfy $h(r, 0)=k(r, 0)=0, h(0, \epsilon)$ $=h(1, \epsilon)=k(0, \epsilon)=k(1, \epsilon)=0$, and $\epsilon$ is a small parameter to be specified later. Asymptotic solutions of (4.1) and (4.2) are sought which deviate from the uniform solution by $O(\epsilon)$ when the parameter $B$ exceeds the critical value $B_{c}=B_{c}(A, D, \vartheta)$ given by (3.14). The parameter $\vartheta$ is held fixed in this procedure. The amount by which $B$ is greater than $B_{c}$ depends on $\epsilon$ in manner to be determined.

The two times to be used in the perturbation calculation are the fast time $t^{*}=t$ and the slow time $\tau=\left(B(\epsilon)-B_{c}\right) t$. The solutions of (4.1) and (4.2) are to be 
given by the asymptotic expansions

$$
\begin{aligned}
& X \sim A+\epsilon u_{1}(r, t, \tau)+\epsilon^{2} u_{2}(r, t, \tau)+\cdots, \\
& Y \sim \frac{B}{A}+\epsilon v_{1}(r, t, \tau)+\epsilon^{2} v_{2}(r, t, \tau)+\cdots,
\end{aligned}
$$

with homogeneous boundary conditions for the $u_{j}$ and $v_{j}$, and initial conditions

$$
\begin{aligned}
& u_{j}(r, 0,0)=\frac{1}{j !} \frac{\partial^{j} h(r, 0)}{\partial \epsilon^{j}}, \\
& v_{j}(r, 0,0)=\frac{1}{j !} \frac{\partial^{j} k(r, 0)}{\partial \epsilon^{j}} .
\end{aligned}
$$

Substituting the expansions (4.3) and (4.4) into equations (4.1) and (4.2), and equating the coefficients of powers of $\epsilon$ to zero, we get a hierarchy of differential equations for the functions $u_{j}(r, t, \tau)$ and $v_{j}(r, t, \tau)$. The equations for $u_{1}$ and $v_{1}$ are just (3.1) and (3.2) with $B=B_{c}$, and the equations for $u_{j}$ and $v_{j}$ with $j>1$ are forced versions of (3.1) and (3.2) with the forcing depending only on the earlier equations in the hierarchy. Then $u_{1}$ and $v_{1}$ are given by the sum

$$
\left[\begin{array}{l}
u_{1} \\
v_{1}
\end{array}\right]=\sum_{n=1}^{\infty}\left\{c_{n}^{+}(\tau) \exp \sigma_{n}^{+} t\left[\begin{array}{c}
\sin n \pi r \\
M_{n}^{+} \sin n \pi r
\end{array}\right]+c_{n}^{-}(\tau) \exp \sigma_{n}^{-} t\left[\begin{array}{c}
\sin n \pi r \\
M_{n}^{-} \sin n \pi r
\end{array}\right]\right\}
$$

Since $B$ is set equal to $B_{c}$ in the perturbation expansion, all the eigenvalues $\sigma_{n}^{ \pm}$ have negative real parts except one, $\sigma_{N}^{+}$, which is equal to zero. (In the exceptional case that $\vartheta, B_{c}$ is one of the intersection points $K_{N}$, both $\sigma_{N}^{+}=0$ and $\sigma_{N+1}^{+}=0$.) Thus all terms in the sum die away exponentially on the fast time scale except the one corresponding to $\sigma_{N}^{+}$, and the slow time variation of the coefficient $c_{N}^{+}(\tau)$ only is important. (In the exceptional case, the slow time variation of $c_{N}^{+}(\tau)$ and $c_{N+1}^{+}(\tau)$, will be important.)

The initial value $c_{N}^{+}(0)$ is found by expanding the initial conditions $u_{j}(r, 0,0)$ and $v_{j}(r, 0,0)$ in series of eigenfunctions. Then, an ordinary differential equation for $c_{N}^{+}(\tau)$ is found by imposing a boundedness condition on $u_{2}$ and $v_{2}$. One takes the inner product of the differential equations for $u_{2}$ and $v_{2}$ with the eigenfunction $\boldsymbol{\psi}_{N}^{+}$, which is the eigenfunction of the boundary value problem adjoint to the one for $u_{1}$ and $v_{1}$ corresponding to the eigenvalue $\overline{\sigma_{N}^{+}}=0$. Then, one integrates from $t=0$ to $t=T$, divides by $T$, and takes the limit as $T \rightarrow \infty$. Since $u_{2}$ and $v_{2}$ are assumed bounded, various terms vanish under this procedure, whereas a condition must be placed on $c_{N}^{+}(\tau)$ to eliminate the others. This condition is a first order ordinary differential equation. For $N$ odd and $B_{c} / A+2 A M_{N}^{+} \neq 0$ this differential equation is quadratic in $c_{N}^{+}$and has the two steady states 0 and $c_{N}^{+}(\infty)$, where

$$
c_{N}^{+}(\infty)=\frac{-B^{\prime}(0)}{(8 / 3 N \pi)\left(B_{c} / A+2 A M_{N}^{+}\right)}
$$

If $c_{N}^{+}(0)$ has the same sign as $c_{N}^{+}(\infty)$, then as $\tau \rightarrow \infty, c_{N}^{+}(\tau)$ tends to the constant limit $c_{N}^{+}(\infty)$, which does not otherwise depend on the initial conditions. If $c_{N}^{+}(0)$ and $c_{N}^{+}(\infty)$ have opposite signs, then $c_{N}^{+}(\tau)$ tends to infinity in a finite time. 
At this stage $\epsilon$ and $B^{\prime}(0)$ may be fixed by the normalization $B-B_{c}=\epsilon$. Then the bifurcating steady state solution for the case $N$ odd and $B_{c} / A+2 A M_{N}^{+} \neq 0$ is

$$
X \sim A-\frac{\left(B-B_{c}\right) \sin N \pi r}{\frac{8}{3 N \pi}\left(\frac{B_{c}}{A}+2 A M_{N}^{+}\right)}+O\left(\left(B-B_{c}\right)^{2}\right)
$$

and

$$
Y \sim \frac{B_{c}}{A}-\frac{\left(B-B_{c}\right) M_{N}^{+} \sin N \pi r}{\frac{8}{3 N \pi}\left(\frac{B_{c}}{A}+2 A M_{N}^{+}\right)}+O\left(\left(B-B_{c}\right)^{2}\right)
$$

The method of construction of the solution (4.6) and (4.7) shows that it is stable, since initial conditions starting near the steady state tend to it. However, if the initial conditions are such that $c_{N}^{+}(0)$ and $c_{N}^{+}(\infty)$ have opposite signs, then the solution does not tend to the steady state (4.6) and (4.7). Presumably (since $c_{N}^{+}(\tau)$ tends to infinity in finite time) it tends to a steady state having finite deviation from the uniform solution.

If $B$ is slightly less than $B_{c}$, so that $B^{\prime}(0)$ is negative, it is possible to go through the same analysis as above and discover that there is a bifurcating steady state, given by (4.6) and (4.7), which is approached as $\tau=\left(B-B_{c}\right) t$ tends to $+\infty$. This steady state must therefore be unstable, as initial conditions starting near it tend away from it as $t$ increases. (Note that $\tau$ tends to $+\infty$ as $t$ tends to $-\infty$ in this case.)

If $N$ is even or $B_{c} / A+2 A M_{N}^{+}=0$, the coefficient of $\left(c_{N}^{+}\right)^{2}$ in the differential equation for $c_{N}^{+}(\tau)$ vanishes. In order to prevent exponential growth of the solution of the resulting linear equation, it is necessary to choose $B^{\prime}(0)=0$. Then, the linear equation is satisfied identically, and in order to obtain a differential equation for $c_{N}^{+}(\tau)$ it is necessary to impose a boundedness condition on $u_{3}$ and $v_{3}$. This is done in the same manner as before. The resulting first order differential equation for $c_{N}^{+}(\tau)$ is cubic in $c_{N}^{+}$and has the three steady states $0, c_{N}^{+}(\infty),-c_{N}^{+}(\infty)$, where

$$
c_{N}^{+}(\infty)=\left(\frac{B^{\prime \prime}(0)}{2 \alpha}\right)^{1 / 2}
$$

If $B_{c} / A+2 A M_{N}^{+}=0$, then $\alpha=-\frac{3}{4} M_{N}^{+}$, while if $N$ is even and $B_{c} / A+2 A M_{N}^{+} \neq 0$, then

$$
\alpha=-\left\{\frac{3}{4} M_{N}^{+}+4 A \int_{0}^{1} \sin ^{2} N \pi r\left(\zeta(r)+M_{N}^{+} \omega(r)\right) d r+\frac{4 B_{c}}{A} \int_{0}^{1} \sin ^{2} N \pi r \omega(r) d r\right\},
$$

where

$$
\omega(r)=\sum_{n \neq N} \frac{\left(B_{c} / A+2 A M_{N}^{+}\right)\left(1+\left(A^{2} / B_{c}\right) M_{n}^{ \pm}\right) \int_{0}^{1} \sin ^{2} N \pi r \sin n \pi r d r}{\left.\frac{1}{2} \sigma_{n}^{ \pm}\left(1-\left(A^{2} / B_{c}\right)\right) M_{n}^{ \pm}\right)^{2}} \sin n \pi r
$$


and

$$
\zeta(r)=\sum_{n \neq N} \frac{\left(B_{c} / A+2 A M_{N}^{+}\right)\left(1+\left(A^{2} / B_{c}\right) M_{n}^{ \pm}\right) \int_{0}^{1} \sin ^{2} N \pi r \sin n \pi r d r}{\frac{1}{2} \sigma_{n}^{ \pm}\left(1-\left(A^{2} / B_{c}\right) M_{n}^{ \pm}\right)^{2}} M_{n}^{ \pm} \sin n \pi r .
$$

A convenient normalization of $\epsilon$ and $B^{\prime \prime}(0)$ is $B-B_{c}=\epsilon^{2}$. Then the bifurcating steady state solutions for the case $N$ even or $B_{c} / A+2 A M_{N}^{+}=0$ are

$$
\begin{aligned}
& X \sim A \pm\left(\frac{B-B_{c}}{\alpha}\right)^{1 / 2} \sin N \pi r+O\left(B-B_{c}\right) \\
& Y \sim \frac{B_{c}}{A} \pm\left(\frac{B-B_{c}}{\alpha}\right)^{1 / 2} M_{n}^{+} \sin N \pi r+O\left(B-B_{c}\right) .
\end{aligned}
$$

If $c_{N}^{+}(0)$ is positive (negative), then the steady state that is approached uses the plus (minus) sign in (4.8) and (4.9).

These results are summarized in the bifurcation diagrams, Figs. 4.1 and 4.2. The "amplitude" is the coefficient of $\sin N \pi r$ in (4.6) or (4.8). Stable branches are indicated by solid lines, and unstable branches by dotted lines.

In the exceptional case that $\vartheta, B_{c}$ is one of the points $K_{N}$, so that both $\boldsymbol{\phi}_{N}^{+}$and $\boldsymbol{\phi}_{N+1}^{+}$are excited, it is necessary to find the slow time variation of the coefficients $c_{N}^{+}(\tau)$ and $c_{N+1}^{+}(\tau)$. This is done in the same manner as before, but it is necessary to take the inner product of the equations for $u_{2}$ and $v_{2}$ with both $\boldsymbol{\psi}_{N}^{+}$and $\boldsymbol{\psi}_{N+1}^{+}$. There results a pair of coupled first order differential equations, quadratic in $c_{N}^{+}$and $c_{N+1}^{+}$. Some initial conditions tend to infinity in a finite time. Again the interpretation is that they actually tend to a steady state having finite deviation from the uniform
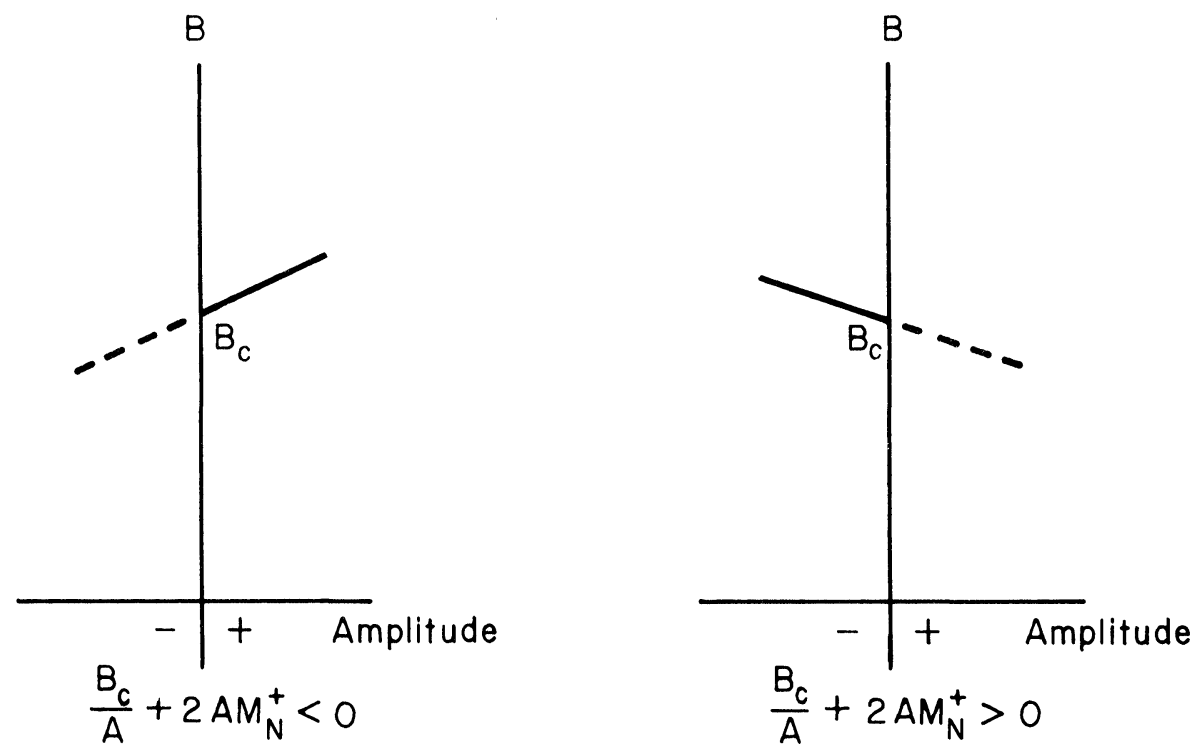

FIG. 4.1. Bifurcation of a nonuniform steady state when $N$ is odd 


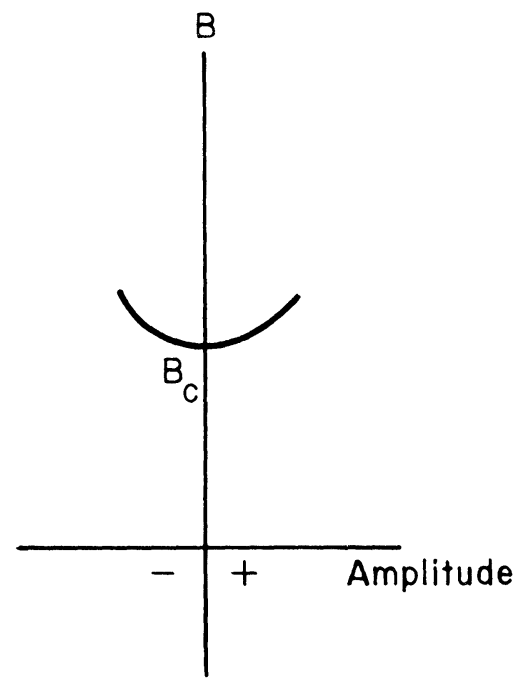

FIG. 4.2. Bifurcation of nonuniform steady states when $N$ is even

state. Other initial conditions may tend to a bifurcating steady state having small deviation from the uniform state.

It is of interest to find how the solution branches of Figs. 4.1 and 4.2 are to be extended to finite amplitude disturbances. This has been done in the limiting case $\vartheta=\infty$; the calculations are given in [1].

5. Possibility of localization. When $A(r)$ is no longer uniformly distributed and the basic steady state $X_{0}(r), Y_{0}(r)$ is slightly unstable, one expects to see the bifurcation of nonuniform states as in $\S 4$. When the point $\vartheta, B$ is sufficiently close to the neutral stability curve, the bifurcating steady state will be to first order a small multiple of the eigenfunction of the linearized stability problem for which the corresponding eigenvalue has the greatest real part. In this section we show that eigenfunctions may have turning points. The apparent localization of the nonuniform disturbance is then due to the fact that the eigenfunction is oscillatory in the middle of the region $0 \leqq r \leqq 1$, but not at the boundaries.

It is assumed that $D \ll 1$. The WKBJ technique is used to investigate

$$
\begin{aligned}
\sigma \phi & =\left[-(B+1)+2 X_{0} Y_{0}\right] \phi+X_{0}^{2} \psi+D \phi^{\prime \prime}, \\
\phi \psi & =\left[B-2 X_{0} Y_{0}\right] \phi-X_{0} \psi+\vartheta D \psi^{\prime \prime},
\end{aligned}
$$

with boundary values $\phi(0)=\phi(1)=\psi(0)=\psi(1)=0$.

We seek solutions to (3.4) and (3.5) of the form

$$
\left[\begin{array}{l}
\phi \\
\psi
\end{array}\right] \sim \exp \frac{i w(r)}{\sqrt{D}}\left\{\left[\begin{array}{l}
f_{0}(r) \\
g_{0}(r)
\end{array}\right]+\sqrt{D}\left[\begin{array}{l}
f_{1}(r) \\
g_{1}(r)
\end{array}\right]+D\left[\begin{array}{l}
f_{2}(r) \\
g_{2}(r)
\end{array}\right]+\cdots\right\} .
$$

Substitution of this expansion into the equations (3.4) and (3.5), and equation of 
coefficients of powers of $\sqrt{D}$ to zero, produces the following hierarchy of equations:

$$
\begin{gathered}
{\left[\begin{array}{cc}
\sigma+w^{\prime 2}+(B+1)-2 X_{0} Y_{0} & -X_{0}^{2} \\
-B+2 X_{0} Y_{0} & \sigma+\vartheta w^{\prime 2}+X_{0}^{2}
\end{array}\right]\left[\begin{array}{l}
f_{0} \\
g_{0}
\end{array}\right]=\left[\begin{array}{l}
0 \\
0
\end{array}\right]} \\
{\left[\begin{array}{cc}
\sigma+w^{\prime 2}+(B+1)-2 X_{0} Y_{0} & -X_{0}^{2} \\
-B+2 X_{0} Y_{0} & \sigma+\vartheta w^{\prime 2}+X_{0}^{2}
\end{array}\right]\left[\begin{array}{l}
f_{1} \\
g_{1}
\end{array}\right]=\left[\begin{array}{c}
i\left(w^{\prime \prime} f_{0}+2 w^{\prime} f_{0}^{\prime}\right) \\
\vartheta i\left(w^{\prime \prime} g_{2}+2 w^{\prime} g_{0}^{\prime}\right)
\end{array}\right] .}
\end{gathered}
$$

Since the system (5.2) is homogeneous, the determinant of the coefficients must be zero in order for a nontrivial solution to exist. A quartic equation for $w^{\prime}(r)$ is obtained, with the solution

$$
\begin{aligned}
2 \vartheta w^{\prime 2}= & -\left\{\vartheta\left[\sigma+(B+1)-2 X_{0} Y_{0}\right]+\sigma+X_{0}^{2}\right\} \\
& \pm\left[\left\{\vartheta\left[\sigma+(B+1)-2 X_{0} Y_{0}\right]+\sigma+X_{0}^{2}\right\}^{2}\right. \\
& \left.-4 \vartheta\left\{\sigma\left[\sigma+(B+1)+X_{0}^{2}-2 X_{0} Y_{0}\right]+X_{0}^{2}\right\}\right]^{1 / 2} .
\end{aligned}
$$

It is also seen from (5.2) that

$$
\left(-B+2 X_{0} Y_{0}\right) f_{0}+\left(\sigma+\vartheta w^{\prime 2}+X_{0}^{2}\right) g_{0}=0,
$$

and that there is some constant $c$ such that

$$
\frac{\sigma+w^{\prime 2}+B+1-2 X_{0} Y_{0}}{B-2 X_{0} Y_{0}}=\frac{X_{0}^{2}}{\sigma+\vartheta w^{\prime 2}+X_{0}^{2}}=c .
$$

Then by adding $c$ times the second equation in (5.3) to the first equation, one obtains the differential equation

$$
w^{\prime \prime}\left(f_{0}+c \vartheta g_{0}\right)+2 w^{\prime}\left(f_{0}+c \vartheta g_{0}\right)^{\prime}=0 .
$$

The phase function $w(r)$ is presumed known from (5.4), so that (5.7) integrates to

$$
\left(f_{0}+c \vartheta g_{0}\right) \sqrt{w^{\prime}}=\text { const., }
$$

where the principal branch of the square root is taken. Then from (5.5),

$$
g_{0}=\frac{\text { const. }}{\sqrt{w^{\prime}}}\left[c \vartheta+\frac{\sigma+\vartheta w^{\prime 2}+X_{0}^{2}}{B-2 X_{0} Y_{0}}\right]^{-1} \text {. }
$$

At this stage, $f_{0}$ and $g_{0}$ are determined up to constants, which are to be determined by imposing the boundary conditions. In general there will be a solution only for certain values of $\sigma$, the eigenvalues of the problem.

However, the asymptotic approximation (5.1) will break down if the expression in brackets in (5.8) should vanish. It is seen from (5.6) that this happens if

$$
2 \vartheta w^{\prime 2}+\vartheta\left[\sigma+(B+1)-2 X_{0} Y_{0}\right]+\sigma+X_{0}^{2}=0 .
$$

Comparing (5.9) and (5.4), one sees that the expansion (5.1) breaks down exactly where the discriminant of (5.4) vanishes. The points where the discriminant of (5.4) vanishes are termed turning points, by analogy with the similar situation in quantum theory. 
It is of particular interest to locate the turning points in the case $\sigma=0$. The value $B_{c}$ of $B$ that corresponds to this value of $\sigma$ will be the branching point for a bifurcating branch of steady states. In this case, the condition that the discriminant of (5.4) vanish is

$$
\left[\vartheta\left[\left(B_{c}+1\right)-2 X_{0} Y_{0}\right]+X_{0}^{2}\right]^{2}=4 \vartheta X_{0}^{2} .
$$

In terms of $X_{0}=A(r)+O(D)$ and $Y_{0}=B_{c} / A(r)+O(D)$, this is

$$
\left[\vartheta\left(-B_{c}+1\right)+A^{2}(r)\right]^{2}=4 \vartheta A^{2}(r)+O(D)
$$

or

$$
B_{c}=1-\frac{2 A(r)}{\sqrt{\vartheta}}+\frac{A^{2}(r)}{\vartheta}+O(\sqrt{D}) .
$$

Thus, if $B_{c}$ is such that there is a value of $r$ that satisfies (5.10), there is a turning point there. Actually, since $A(r)$ is a catenary, there will be two turning points $r_{1}$ and $r_{2}$, symmetric with respect to $r=\frac{1}{2}$. It is seen from (5.4) that for $r_{1}<r<r_{2}$, the phase $w(r)$ is complex so that the exponential $\exp i D^{-1 / 2} w(r)$ is oscillatory in that region. For $r<r_{1}$ and $r>r_{2}, w^{\prime 2}$ is negative so that exp $i D^{-1 / 2} w(r)$ is not oscillatory. Thus the bifurcating steady states show spatial oscillations in the middle of the slab, but not at the edges. If $B_{c}$ lies above $\left(1-A_{\max } / \sqrt{\theta}+O(\sqrt{D})\right)^{2}$, then $w(r)$ is complex across the entire interval $0 \leqq r \leqq 1$, so that the localization of the disturbance disappears.

Acknowledgment. James A. Boa would like to thank Professor J. B. Keller for some illuminating discussions.

\section{REFERENCES}

[1] JAMES A. BOA, Multiple steady states in a model biochemical reaction, Studies in Applied Mathematics, 54 (1975), pp. 9-15.

[2] Britton Chance, E. Kendall Pye, Amal K. Ghosh and Benno Hess, Biological and Biochemical Oscillators, Academic Press, New York, 1973.

[3] M. HERSCHKOWITZ-KAUFMAN AND G. NiCOLIS, Localized spatial structures and nonlinear chemical waves in dissipative systems, J. Chem. Phys., 56 (1972), pp. 1890-1895.

[4] Stanley Kogelman AND JOSEPH B. Keller, Transient behavior of unstable nonlinear systems with applications to the Bénard and Taylor problems, this Journal, 20 (1971), pp. 619-637.

[5] В. J. Mаткоwsкy, A simple nonlinear dynamic stability problem, Bull. Amer. Math. Soc., 76 (1970), pp. 620-625.

[6] G. NicOlis AND J. F. G. AuCHMUTY, Dissipative structures, catastrophes and pattern formation: a bifurcation analysis, Proc. Nat. Acad. Sci., 71 (1974), pp. 2748-2751.

[7] ARTHUR T. WINFREE, Scroll-shaped waves of chemical activity in three dimensions, Science, 181 (1973), pp. 937-939.

[8] A. M. Zhabotinsky And A. N. Zaikin, Autowave processes in a distributed chemical system, J. Theor. Biol., 40 (1973), pp. 45-61.

[9] JAmes A. BOA, A model biochemical reaction, Ph.D. thesis, California Institute of Technology, Pasadena, 1974. 